\title{
The relationship with parent associations
}

\author{
Novegno Federica
}

Received: 25 February 2014 / Accepted: 25 February 2014 / Published online: 7 March 2014

(C) Springer-Verlag Berlin Heidelberg 2014

In recent years, the relationship with parent associations has gained a significant influence on the fulfillment of the clinical pathway shared by the patients, their relatives, and the medical staff. They represent nonprofit organizations, which devote their activities to support people who suffer from a disease (Fig. 1).

The ultimate purpose is the improvement of treatment, quality of life, and long-term outlook for neurosurgical patients, more in general, for patients affected by a pathology, who require both the most appropriate medical care as well as psychological, sociological, and economic support.

In a schematic view, the medical staff offers its aid of expertise and experience, achieved with medical education and constant updating and supported by technical innovations and research improvement; and the family and the parents, who hold the hand of the patients at their best, deal every day with the fear of what they do not know or what they do not understand completely, and are involved in the socioeconomic assistance to the patient.

The relationships among these characters are often burdened by incomprehension, disinformation, and the work of the medical staff often limited by lack of resources or by slow research improvement.

Parent associations arise from the need to fill the whole series of gaps which lie on these relations, so that patient care may be improved, both qualitatively and quantitatively.

Therefore, the objective of the associations should be to stimulate and encourage initiatives to improve and develop the technical, organizational, and social fields to relieve the discomfort to the patient and his family caused by illness,

N. Federica $(\bowtie)$

Department of Neurosurgery, University of Rome "Tor Vergata",

V.le Oxford 81, 00133 Rome, Italy

e-mail: federicanovegno@hotmail.it supporting the clinical centers that deal with issues related to the disease.

The scope is broad and includes a contribution to different fields, namely social assistance, information, education, research, collaboration, and international cooperation.

The social assistance is carried out at different levels: for the patient, providing psychological assistance, and in particular, for children patients, concurring to set up familiar spaces in the medical ward, managing the playing areas and buying toys and educational materials, organizing parties for children in the hospital, and recreational outing and offering clown therapy, which represent a whole set of actions aimed at relieving the hospital stay and the way at coping with the disease. For the relatives, the social assistance is carried out with the management of the daycare houses, providing economical support, information service and easy terms for journeys, managing direct relationship with public or private specific medical centers, and developing operative relationships with public institutions at the local and national levels and worldwide.

Information has a threefold function: firstly, giving true knowledge to the families about the disease, research progresses, available therapies, and so on by means of organizing conferences, lectures with specialists, providing brochures, and illustrative material, and in particular, referring to the correct information on what is the actual progress of research and the available therapies, refuting the distorting point of view of noncompetent people who often offers miraculous therapies with no scientific validation. Secondly, acquiring information about medical and economic assistance of the public institutions and counseling for legal acknowledgement of disability, tax exemption, and economical contribution for attending school is of primary importance for families as well. Thirdly, information is also intended to sensitize public awareness and public and private institutions about the social, medical, and therapeutic problems related to specific pathologies. 
Fig. 1 Logos of some of the parent association working worldwide

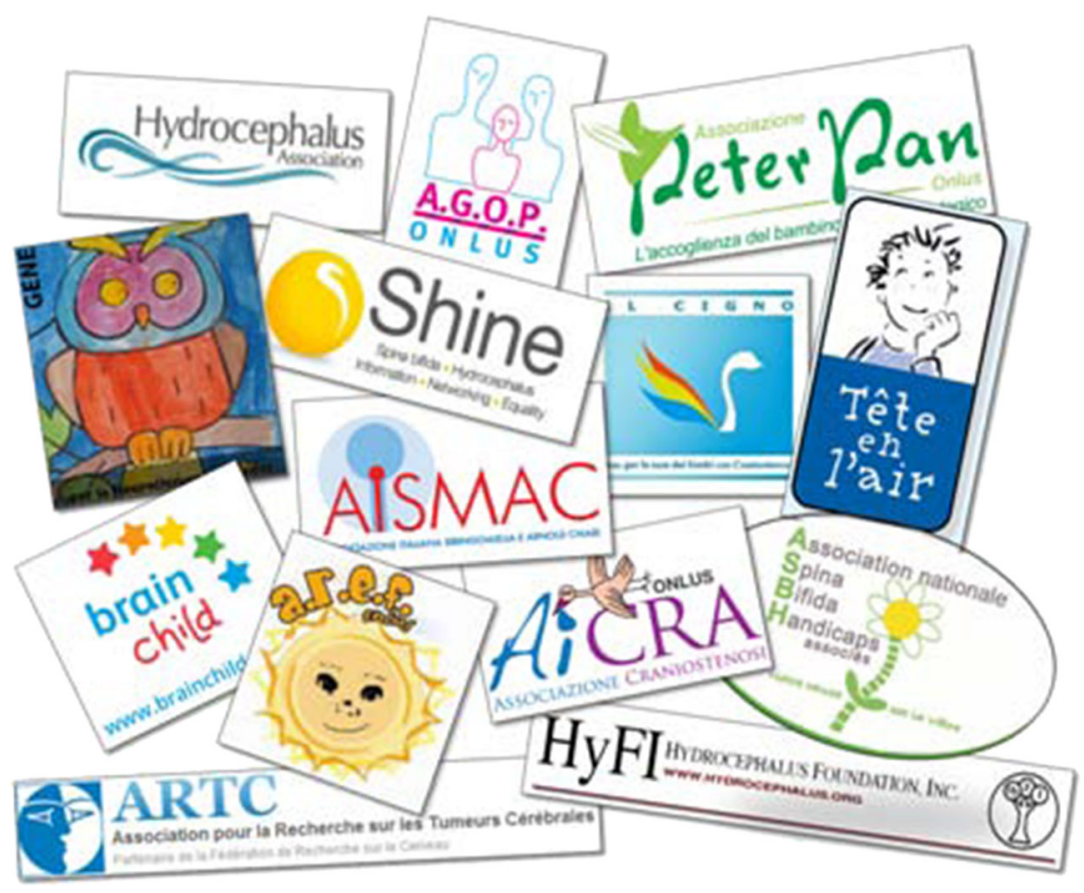

Patients with medical diseases are not only a pathology to treat; the treatment you can best give them would offer better results and wellness to the patients. However, this aspect may be achieved only by investing in education and research. In fact, the role of parent associations is also supporting constant retraining of doctors, nurses, and all the people involved in medical care, by means of connections with specialized national and international centers; supporting the organization of national and international congresses; and awarding scholarship grants. Moreover, they are frequently involved in promoting clinical and molecular research with the realization of innovative therapeutic strategies, collecting funding for specific projects, allowing for the collaboration among the researchers, and creating a direct relationship between them and the patients and their families. They support the participation of researchers into international projects also for new clinical testing, allowing for the improvement of knowledge of new therapies.

To further improve the achievements and increase the possibility of intervention, parent associations may collabo- rate with other entities and nonprofit institutions having similar purposes, even through affiliation and promotion of federations or other associative commitment. In some cases, the activities of parent association may reach foreign countries, promoting an international cooperation with the aim of an independent and sustainable development through transfer of skills, protocols, and technologies compatible with the environment, as well as through prevention and information.

To summarize, the primary objective of parent associations is the relief of the sense of loneliness of the people and their families who deal with illness every day, achieving a horizontal communication, converting the care needs into welfare rights, and providing an updated resource map.

For this and more, we need them, and we thank them.

Acknowledgments We wish to thank the following associations for having given permission for logo publication: Hydrocephalus, A.G.O.P, Peter Pan, GENE Federation, Shine, Il Cigno, Tete en l'air, Brain Child, A.R.E.F., AISMAC, AICRA, ASBH, ARTC, and HyFy. 